\title{
A NOTE ON WRONSKIANS AND THE ABC THEOREM IN FUNCTION FIELDS OF PRIME CHARACTERISTIC
}

\author{
Julie Tzu-Yueh Wang \\ Institute of Mathematics \\ Academia Sinica \\ Nankang, Taipei 11529 \\ Taiwan, R.O.C.
}

May 14, 1998

\begin{abstract}
We provide a sharp bound for the order sequence of Wronskians. We also give another proof of the truncated second main theorem over function fields which is a generalization of the ABC theorem due to Mason, Voloch, Brownawell and Masser, Noguchi and the author.
\end{abstract}

\section{INTRODUCTION}

Let $C$ be an irreducible nonsingular projective algebraic curve of genus $g$ defined over an algebraically closed field $k$ of characteristic $p$. Let $K$ be the function field of $C$. Suppose that $t$ is a local parameter of a point $P$ of $C$, i.e. $v_{P}(t)=1 . K$ is then a finite separable field extension of $k(t)$.(cf. [Si], Chapter II, Proposition 1.4) Recall that the Hasse derivatives $D_{t}^{(i)}$ is defined on $k(t)$ by $D_{t}^{(i)}\left(t^{j}\right)=\left(\begin{array}{l}j \\ i\end{array}\right) t^{j-i}$, and then can be naturally extended to $K(\mathrm{cf}$. $[\mathrm{S}-\mathrm{V}])$. The Hasse derivatives satisfy the following:

Proposition 1.1. (a) $D_{t}^{(i)}(z w)=\sum_{j=0}^{i} D_{t}^{(j)}(z) D_{t}^{(i-j)}(w), \quad \forall z, w \in K$.

(b) $D_{t}^{(i)} D_{t}^{(j)}=\left(\begin{array}{c}i+j \\ i\end{array}\right) D_{t}^{i+j}$.

Let now $p>0$. Following Garcia and Voloch's notation in [G-V], we let $K_{m}=$ $\left\{x \in K \mid D_{t}^{(i)}(x)=0\right.$ for $\left.1 \leq i<p^{m}\right\}$ and let $K_{\infty}=\left\{x \in K \mid D_{t}^{(i)}(x)=0\right.$ for $\left.i \geq 1\right\}$.

Proposition 1.2. For all $m \geq 1, K_{m}$ is a field. Moreover, $D_{t}^{\left(p^{m}\right)}$ is a derivation on $K_{m}$ and $K_{m+1}=\left\{x \in K_{m} \mid D_{t}^{\left(p^{m}\right)}(x)=0\right\}$.

Proof. See $[\mathrm{G}-\mathrm{V}]$, Proposition 1. 
Remark. $K_{m}=k K^{p^{m}}$ and $K_{\infty}=k$ (See [G-V]).

Throughout the paper $x_{0}, \ldots, x_{n}$ will denote elements of $K$.

Garcia and Voloch also proved the following (cf. [G-V])

Theorem A. $x_{0}, \ldots, x_{n}$ are linearly independent over $K_{m}$ if and only if there exist integers $0=\epsilon_{0}<\cdots<\epsilon_{n}<p^{m}$ with $\operatorname{det}\left(D_{t}^{\left(\epsilon_{i}\right)}\left(x_{j}\right)\right) \neq 0$

In [Wa1], the author gave an upper bound for each $\epsilon_{i}$ by showing the following:

Theorem B. If $x_{0}, \ldots, x_{n}$ are linearly independent over $K_{m}$, then there exist integers $0=\epsilon_{0}<\epsilon_{1}<\ldots<\epsilon_{n}$ with $\epsilon_{i} \leq i p^{m-1}$ such that

$$
\operatorname{det}\left(D_{t}^{\left(\epsilon_{i}\right)} x_{j}\right)_{0 \leq i, j \leq n} \neq 0
$$

This bound is the best possible when $n<p$. For example,

Example. Let $K=k(t)$ and $p>n$. Let $x_{i}=t^{i p^{(m-1)}}, 0 \leq i \leq n$, where $m$ is a positive integer. Then $x_{0}, \ldots, x_{n}$ are linearly independent over $K^{p^{m}}$, and $\epsilon_{i}=i p^{(m-1)}$.

When $n \geq p$, this bound is clearly not the best possible. We will see later that the field extension degree $\left[K: K^{p^{\alpha}}\right]=p^{\alpha}$. Therefore, when $p^{\alpha} \leq n<p^{\alpha+1}$ it only makes sense to consider the case where $x_{0}, \ldots, x_{n}$ are linearly independent over $K^{p^{m}}$ for $m \geq \alpha+1$. In Section 2, we will locate the best possible bound for each $\epsilon_{i}$ in the general case. The bound on $\epsilon_{i}$ is closely related to the theory of Weierstrass points in positive characteristic.(cf. $[\mathrm{G}-\mathrm{V}],[\mathrm{S}-\mathrm{V}]$, etc.)

In [Wa1], the author used the previous theorem and some results of $[\mathrm{S}-\mathrm{V}]$ on Weierstrass points to prove the truncated second main theorem of function fields of any characteristic which is a generalization of the ABC Theorem due to Mason, Voloch, and Brownawell and Masser. The truncated second main theorem has many applications in function field Diophantine geometry. For example, it was used by the author to study the $S$-integral points on projective spaces minus hyperplanes.(cf. [Wa1], [Wa2], [Wa3], and [Wa4]) In Section 3, we will include another proof which is in the flavor of Nevanlinna theory and only involves Wronskians. We should also mention here that Noguchi(cf. [No]) also used techniques from Nevanlinna theory to show a truncated second main theorem for function fields of higher dimension with characteristic zero. 
Ackowlegements. This note is motivated by some questions about the $\mathrm{ABC}$ theorem and the theory of Weierstrass points that arose during the $\mathrm{ABC}$ workshop of the 1998 Arizona Winter School. The author would like to thank Professor W. McCallum for suggestions on the proof of Lemma 2.1. The author also wishes to thank Professor J. F. Voloch for some helpful comments.

\section{WRONSKIANS}

We first formulate some results to understand the structure of the vector space spanned by $\left\{x_{0}, \ldots, x_{n}\right\}$ over $K^{p^{\gamma}}, \gamma \geq 1$.

Lemma 2.1. Let $u$ and $m$ be nonnegative integers. Then the field extension degree of $\left[K^{p^{m}}, K^{p^{m+u}}\right]$ is $p^{u}$. Furthermore, $\left\{t^{i p^{m}} \mid 0 \leq i \leq p^{u}-1\right\}$ is a set of basis for the vector space $K^{p^{m}}$ over $K^{p^{m+u}}$.

Proof. To show the field extension degree of $\left[K^{p^{m}}, K^{p^{m+u}}\right]$ is $p^{u}$, we first prove that $\left[K: K^{p}\right]=p$. It then follows similarly that $\left[K^{p^{m}}: K^{p^{m+1}}\right]=p$. Therefore $\left[K^{p^{m}}, K^{p^{m+u}}\right]=p^{u}$.

Since the function field $K$ is a finite separable field extension over $k(t)$, there exists an element $y \in K$ such that $K=k(t, y)$. Furthermore, we may assume that $y$ satisfies a monic minimal polynomial $f(Y) \in k(t)[Y]$. Then we have

$$
\begin{aligned}
& {[k(t, y): k(t)]\left[k(t): k\left(t^{p}\right)\right]} \\
& \left.=[k(t, y)]: k\left(t^{p}\right)\right] \\
& =\left[k(t, y): k\left(t^{p}, y^{p}\right)\right]\left[k\left(t^{p}, y^{p}\right): k\left(t^{p}\right)\right] .
\end{aligned}
$$

Because that $\left[k(t): k\left(t^{p}\right)\right]=p$, it remains to show that $[k(t, y): k(t)]=\left[k\left(t^{p}, y^{p}\right)\right.$ : $\left.k\left(t^{p}\right)\right]$.

Suppose that $f(Y)=Y^{r}+a_{n-1}(t) Y^{r-1}+\cdots+a_{0}(t)=\tilde{f}(t, Y)$, where $a_{i}(t) \in$ $k(t)$. We raise each coefficient of $a_{i}(t)$ to its $p$-th power and denote it by $a_{i}^{p}(t)$. Let $\tilde{f}^{p}(t, y)=Y^{r}+a_{n-1}^{p}(t) Y^{r-1}+\cdots+a_{0}^{p}(t)$. Then $\tilde{f}^{p}\left(t^{p}, y^{p}\right)=(\tilde{f}(t, y))^{p}=0$. Therefore, $\left[k\left(t^{p}, y^{p}\right): k\left(t^{p}\right)\right] \leq r$. Since $k$ is an algebraically closed field, $k=k^{p}$. Therefore if $\tilde{f}^{p}\left(t^{p}, Y^{p}\right)$ is reducible over $k\left(t^{p}\right)$, then $f(t, Y)$ will be reducible in $k(t)[Y]$ which gives a contradiction. Hence $\left[k\left(t^{p}, y^{p}\right): k\left(t^{p}\right)\right]=r=[k(t, y): k(t)]$.

Similarly, one can show that $\left[K^{p^{m}}: K^{p^{m+1}}\right]=p$. Therefore $\left[K^{p^{m}}, K^{p^{m+u}}\right]=p^{u}$.

One can easily see that $\operatorname{det}\left(D_{t}^{\left(i p^{m}\right)} t^{j p^{m}}\right)_{0 \leq i, j \leq p^{u}-1} \neq 0$, so the second part of the assertion follows directly from the Theorem A and the first assertion. 
Lemma 2.2. Suppose $p^{\alpha} \leq n<p^{\alpha+1}$ for some nonnegative integer $\alpha$, and let $\beta$ be a nonnegative integer less than or equal to $\alpha$. If $x_{0}, \ldots, x_{n}$ are linearly independent over $K^{p^{\alpha+s}}$ for some positive integer $s$, then the dimension of the vector space spanned by $x_{0}, \ldots, x_{n}$ over $K^{p^{\beta+s}}$ is strictly greater than $p^{\beta}$.

Proof. Suppose $r+1$ is the dimension of the vector space spanned by $x_{0}, \ldots, x_{n}$ over $K^{p^{\beta+s}}$. Without loss of generality, we can assume that $x_{0}, \ldots, x_{r}$ are linearly independent over $K^{p^{\beta+s}}$. By Lemma 2.1, $\left\{t^{i p^{\beta+s}} \mid 0 \leq i \leq p^{\alpha-\beta}-1\right\}$ is a set of basis for the vector space $K^{p^{\beta+s}}$ over $K^{p^{\alpha+s}}$. Then the set

$$
\left\{t^{i p^{\beta+s}} x_{j} \mid 0 \leq i \leq p^{\alpha-\beta}-1,0 \leq j \leq r\right\}
$$

is linearly independent over $K^{p^{\alpha+s}}$. Since $x_{j}, r+1 \leq j \leq n$, is a linear combination of $x_{0}, \ldots, x_{r}$ over $K^{p^{\beta+s}}$, the dimension of the vector space spanned by $\left\{t^{i p^{\beta+s}} x_{j} \mid 0 \leq i \leq p^{\alpha-\beta}-1,0 \leq j \leq r\right\}$ over $K^{p^{\alpha+s}}$ is no less than the dimension of the vector space spanned by $\left\{x_{0}, \ldots, x_{n}\right\}$ over $K^{p^{\alpha+s}}$. Therefore, $p^{\alpha-\beta}(r+1) \geq n+1>p^{\alpha}$. Hence $r+1>p^{\beta}$.

We will formulate an upper bound on $\epsilon_{i}$ according to the dimension of each vector space spanned by $\left\{x_{0}, \ldots, x_{n}\right\}$ over $K^{p^{\gamma}}$ for $\gamma \geq 0$. Later, we will offer some examples to show that this formulation is necessary.

Theorem 1. Suppose that $p^{\alpha} \leq n<p^{\alpha+1}$ and $m \geq \alpha+1$. Let the dimension of the vector space spanned by $x_{0}, \ldots, x_{n}$ over $K^{p^{\gamma}}$ be $l_{\gamma}+1$ for $0 \leq \gamma \leq m$. Let $0=\gamma_{0}<\gamma_{1}<\cdots<\gamma_{u} \leq m$ be a sequence of integers between 0 and $m$ such that $0=l_{\gamma_{0}}<l_{\gamma_{1}}<\cdots<l_{\gamma_{u}}=n$ and $l_{\gamma_{\delta}}=l_{\gamma_{\delta}+1}=\cdots=l_{\gamma_{\delta+1}-1}$. Then $x_{0}, \ldots, x_{n}$ are linearly independent over $K^{p^{m}}$ if and only if there exist integers $0=\epsilon_{0}<\epsilon_{1} \cdots<\epsilon_{n}$ with

$$
p^{\gamma_{\delta}-1}+s-1 \leq \epsilon_{l_{\gamma_{\delta}-1}+s} \leq \min \left\{s p^{\gamma_{\delta}-1}, p^{\gamma_{\delta}}-1\right\}, \quad 1 \leq s \leq l_{\gamma_{\delta}}-l_{\gamma_{\delta-1}},
$$

such that $\operatorname{det}\left(D_{t}^{\left(\epsilon_{i}\right)} x_{j}\right)_{0 \leq i, j \leq n} \neq 0$.

Remark 2.1. By Lemma 2.2, $l_{m-\alpha+i} \geq p^{i}$.

Remark 2.2. It is easy to see that in general one has $\epsilon_{i} \leq i p^{m}$. Therefore Theorem 1 is stronger than the Theorem B.

Remark 2.3. When the characteristic of $k$ is $0, \epsilon_{i}=i$. 
Corollary. $\epsilon_{l_{\gamma_{-1}+1}}=p^{\gamma_{\delta}-1}$, for $\delta=1,2, \ldots, u$.

Proof. It follows easily from the inequalities in Theorem 1.

Proof of Theorem 1. The "if " part comes from Theorem A. Therefore, we only need to show the other direction.

Without loss of generality, we may assume that $x_{0}, \ldots, x_{l_{\gamma \delta}}$ are linearly independent over $K^{p^{\gamma \delta}}$. By definition of $\gamma_{\delta}, x_{0}, \ldots, x_{l_{\gamma_{-1}+1}}$ are linearly dependent over $K^{p^{\gamma^{-1}}}$. Therefore, by Theorem A we have $\epsilon_{l_{\gamma_{-1}+1}} \geq p^{\gamma_{\delta}-1}$. Hence, $\epsilon_{l_{\gamma_{\delta-1}+s}}>$ $\epsilon_{l_{\gamma_{\delta-1}+1}}+s-1 \geq p^{\gamma_{\delta}-1}+s-1$. This proves one side of the inequality.

For the other side of the inequality, we will prove it by induction. Since $x_{0}, \ldots, x_{l_{\gamma_{1}}}$ are linearly independent over $K^{p^{\gamma_{1}}}$, from Theorem B there exist $0=\epsilon_{0}<\epsilon_{1} \cdots<$ $\epsilon_{l_{\gamma_{1}}}$ with $\epsilon_{i} \leq i p^{\gamma_{1}-1}$ such that $\operatorname{det}\left(D_{t}^{\left(\epsilon_{i}\right)} x_{j}\right)_{0 \leq i, j \leq l_{\gamma_{1}}} \neq 0$. Together with Theorem A, we conclude

$$
\epsilon_{i} \leq \min \left\{i p^{\gamma_{1}-1}, p^{\gamma_{1}}-1\right\}, \quad \text { for } 1 \leq i \leq l_{\gamma_{1}}
$$

The proof will be completed by two induction steps. First we show that if the theorem is true for $n=l_{\gamma_{\beta}}, 0 \leq \beta<u$, then it is true for $n=l_{\gamma_{\beta}}+1$. We then show that if the theorem is true for $n=l_{\gamma_{\beta}}+s$, then it is true for $n=l_{\gamma_{\beta}}+s+1$, where $1 \leq s<l_{\gamma_{\beta+1}}-l_{\gamma_{\beta}}$.

Now suppose that the theorem is true for $n=l_{\gamma_{\beta}}$ and the conclusion of the theorem does not hold for $n=l_{\gamma_{\beta}}+1$. Then the vectors

$$
\left(x_{j}, D_{t}^{(1)} x_{j}, D_{t}^{(2)} x_{j}, \ldots, D_{t}^{\left(p^{\gamma_{\beta+1}}-1\right)} x_{j}\right), \quad 0 \leq j \leq l_{\gamma_{\beta}}+1
$$

are linearly dependent over $K$. Then there exist $a_{0}, \ldots, a_{l_{\gamma_{\beta}}+1} \in K$ such that

$$
\sum_{j=0}^{l_{\gamma_{\beta}}+1} a_{j} D_{t}^{(i)} x_{j}=0 \quad \text { for } 0 \leq i \leq p^{\gamma_{\beta+1}-1} .
$$

Since $x_{0}, \ldots, x_{l_{\gamma_{\beta}}}$ are linearly independent over $K^{p^{\gamma_{\beta}}}$, by the induction hypothesis we have that the $l_{\gamma_{\beta}}+1$ vectors above, for $0 \leq j \leq l_{\gamma_{\beta}}$, are linearly independent over $K$. Hence $a_{l_{\gamma_{\beta}}+1} \neq 0$ and, without loss of generality, we can assume that $a_{l_{\gamma_{\beta}}+1}=1$. We will show that $a_{j} \in K^{p^{\gamma_{\beta}+1}}$, for $0 \leq j \leq l_{\gamma_{\beta}}$. Then we have $x_{0}, \ldots, x_{l_{\gamma_{\beta}}+1}$ are linearly dependent over $K^{p^{\gamma_{\beta+1}}}$ which will give a contradiction that completes this step of induction proof. 
To prove that $a_{j} \in K^{p^{\gamma_{\beta+1}}}$, by Proposition 1.2, it suffices to show that $D_{t}^{(r)} a_{j}=0$ for $r=1, p, p^{2}, \ldots, p^{\gamma_{\beta+1}-1}$. We will prove it by induction. For $r=1$, applying $D_{t}$ to $(2.1)$, we have

$$
(i+1) \sum_{j=0}^{l_{\gamma_{\beta}}+1} a_{j} D_{t}^{(i+1)} x_{j}+\sum_{j=0}^{l_{\gamma_{\beta}}} D_{t} a_{j} D_{t}^{(i)} x_{j}=0 .
$$

It follows from (2.1) that

$$
\sum_{j=0}^{l_{\gamma_{\beta}}} D_{t} a_{j} D_{t}^{(i)} x_{j}=0, \quad \text { for } 0 \leq i \leq p^{\gamma_{\beta+1}-1}-1 .
$$

Since the $l_{\gamma_{\beta}}+1$ vectors above (i.e. for $0 \leq j \leq l_{\gamma_{\beta}}$ ) are linearly independent over $K$ we get $D_{t} a_{j}=0$ for $0 \leq j \leq l_{\gamma_{\beta}}$.

Assume that $D_{t}^{\left(p^{\nu}\right)} a_{j}=0$ for $\nu=0,1, \ldots, r-1<\gamma_{\beta+1}-1$ and for $0 \leq j \leq$ $l_{\gamma_{\beta}}$. Then we have $a_{j} \in K^{p^{r}}$. Therefore, $D_{t}^{(b)} a_{j}=0$ for $1 \leq b \leq p^{r}-1$ and for $0 \leq j \leq l_{\gamma_{\beta}}$. Apply $D_{t}^{(b)}, 1 \leq b \leq p^{r}-1$, to (2.1) for $i=p^{\gamma_{\beta+1}-1}$, i.e. $\sum_{j=0}^{l_{\gamma_{\beta}}+1} a_{j} D_{t}^{\left(p^{\gamma_{\beta+1}-1}\right)} x_{j}=0$. Then we have

$$
\left(\begin{array}{c}
p^{\gamma_{\beta+1}-1}+b \\
b
\end{array}\right) \sum_{j=0}^{l_{\gamma_{\beta}+1}} a_{j} D_{t}^{\left(p^{\gamma_{\beta+1}-1}+b\right)} x_{j}=0 .
$$

Since $\left(\begin{array}{c}p^{\gamma_{\beta+1}-1}+b \\ b\end{array}\right) \neq 0 \bmod p$,

$$
\sum_{j=0}^{l_{\gamma_{\beta}}+1} a_{j} D_{t}^{(i)} x_{j}=0, \quad \text { for } 1 \leq i \leq p^{\gamma_{\beta+1}-1}+p^{r}-1 .
$$

Applying the operator $D_{t}^{p^{r}}$ to $(2.1)$ for $0 \leq i \leq p^{\gamma_{\beta+1}-1}-1$, we then have

$$
\sum_{j=0}^{l_{\beta}} D_{t}^{\left(p^{r}\right)} a_{j} D_{t}^{(i)} x_{j}=0, \quad \text { for } 1 \leq i \leq p^{\gamma_{\beta+1}-1}-1 .
$$

Since the $l_{\gamma_{\beta}}+1$ vectors above (i.e. for $0 \leq j \leq l_{\gamma_{\beta}}$ ) are linearly independent over $K$ we get $D_{t}^{\left(p^{r}\right)} a_{j}=0$ for $j=0,1, \ldots, l_{\gamma_{\beta}}$. This completes the first step of the induction proof.

Next, we will show that if the theorem is true for $n=l_{\gamma_{\beta}}+s$, then it is true for $n=l_{\gamma_{\beta}}+s+1,1 \leq s<l_{\gamma_{\beta+1}}-l_{\gamma_{\beta}}$. If $(s+1) p^{\gamma_{\beta+1}-1} \geq p^{\gamma_{\beta+1}}-1$, then by Theorem A the theorem holds. Therefore, we may assume that $(s+1) p^{\gamma_{\beta+1}-1}<$ 
$p^{\gamma_{\beta+1}}-1$. Suppose that the theorem is true for $n=l_{\gamma_{\beta}}+s$, and it does not hold for $n=l_{\gamma_{\beta}}+s+1,1 \leq s<l_{\beta+1}-l_{\beta}$. Similar to the previous argument, we have

$$
\sum_{j=0}^{l_{\gamma_{\beta}}+s+1} a_{j} D_{t}^{(b)} x_{j}=0 \quad \text { for } 0 \leq b \leq(s+1) p^{\gamma_{\beta+1}-1}
$$

Similarly, we can assume that $a_{l_{\gamma_{\beta}}+s+1}=1$. It then suffices to show that $D_{t}^{\left(p^{\nu}\right)} a_{j}=$ 0 for $\nu=1,2,3, \ldots, \gamma_{\beta+1}-1$. We will do it by induction. Suppose $D_{t}^{(\nu)} a_{j}=0$ for $\nu=1,2,3, \ldots, r-1<\gamma_{\beta+1}-1$. Applying the operator $D_{t}^{\left(p^{r}\right)}$ to (2.4) for $0 \leq b \leq s p^{\gamma_{\beta+1}-1}$, we have

$$
\sum_{j=0}^{l_{\gamma_{\beta}}+s} D_{t}^{\left(p^{r}\right)} a_{j} D_{t}^{(b)} x_{j}=0 \quad \text { for } 0 \leq b \leq s p^{\gamma_{\beta+1}-1} .
$$

By the induction hypothesis, the $l_{\gamma_{\beta}}+s+1$ vectors above (i.e. $0 \leq j \leq l_{\gamma_{\beta}}+s$ ) are linearly independent over $K$. Therefore, $D_{t}^{\left(p^{r}\right)} a_{j}=0$ for $j=0,1, \ldots, l_{\gamma_{\beta}}$. Inductively, we have $D_{t}^{(\nu)} a_{j}=0$ for $\nu=1,2,3, \ldots, p^{\gamma_{\beta+1}-1}$. This completes the second step of the induction proof. Therefore the proof for this theorem is completed.

We provide the following examples to show that the results is indeed the best possible and the decomposition on each $K^{p^{\gamma}}$ is necessary.

Example 1. Let $K=k(t), p=3$, and $n=10$. Let $x_{i}=t^{i}$ for $0 \leq i \leq 9$, and let $x_{10}=t^{18}$. Then $x_{0}, \ldots, x_{10}$ are linearly independent over $K^{27}$. In this case, $\alpha=2$, $m=3, l_{0}=0, l_{1}=2, l_{2}=8$, and $l_{3}=9 ; \gamma_{0}=0, \gamma_{1}=1, \gamma_{2}=2$, and $\gamma_{3}=3$. We have $\epsilon_{i}=i$ for $0 \leq i \leq 9$, and $\epsilon_{10}=18$.

Example 2. Let $K=k(t), p=3$, and $n=4$. Let $x_{0}=1, x_{1}=t^{3}, x_{2}=t^{6}$, $x_{3}=t^{9}, x_{4}=t^{18}$. Then $x_{0}, \ldots, x_{4}$ are linearly independent over $K^{27}$. In this case, $\alpha=1, m=3, l_{0}=0, l_{1}=0, l_{2}=2$, and $l_{3}=4 ; \gamma_{0}=0, \gamma_{1}=2$, and $\gamma_{2}=3$. We have $\epsilon_{1}=3, \epsilon_{2}=6, \epsilon_{3}=9, \epsilon_{4}=18$.

Example 3. Let $K=k(t), p=3$, and $n=3$. Let $x_{0}=1, x_{1}=t^{3}, x_{2}=t^{9}$, $x_{3}=t^{18}$. Then $x_{0}, \ldots, x_{3}$ are linearly independent over $K^{27}$. In this case, $\alpha=1$, $m=3, l_{0}=0, l_{1}=0, l_{2}=1$, and $l_{3}=3 ; \gamma_{0}=0, \gamma_{1}=2$, and $\gamma_{2}=3$. We have $\epsilon_{1}=3, \epsilon_{2}=9, \epsilon_{3}=18$. 


\section{The Proof of the Truncated Second Main Theorem}

In this section we will give another proof for the truncated second main theorem in the flavor of Nevanlinna theory. The method which we are going to use here is basically the argument from [B-M] and [La](p.220), but we replace the ordinary higher derivatives by the Hasse derivatives in order to deal with function fields of positive characteristic.

We will need some basic propositions.

Proposition 3.1. (a) If $g_{i}=\sum a_{i j} x_{j}$ with $\left(a_{i j}\right) \in G L_{n+1}(k)$, then

$$
\operatorname{det}\left(D_{t}^{\epsilon_{i}} g_{j}\right)=\operatorname{det}\left(a_{i j}\right) \operatorname{det}\left(D_{t}^{\epsilon_{i}} x_{j}\right)
$$

(b) If $h \in K$, then

$$
\operatorname{det}\left(D_{t}^{\epsilon_{i}} h x_{j}\right)=h^{n+1} \operatorname{det}\left(D_{t}^{\epsilon_{i}} x_{j}\right)
$$

(c) If $x$ is another separating variable, then

$$
\operatorname{det}\left(D_{x}^{\epsilon_{i}} x_{j}\right)=\left(\frac{d t}{d x}\right)^{\epsilon_{0}+\epsilon_{1} \ldots+\epsilon_{n}} \operatorname{det}\left(D_{t}^{\epsilon_{i}} x_{j}\right) .
$$

Proof. See $[\mathrm{S}-\mathrm{V}]$, Proposition 1.4

Proposition 3.2. Let $t_{P}$ be a local parameter of a point $P \in C$. If $x$ be a nonzero element in $K$, then

$$
v_{P}\left(\frac{D_{t_{P}}^{i} x}{x}\right) \geq \begin{cases}-i, & \text { if } v_{P}(x) \neq 0 \\ 0, & \text { if } v_{P}(x)=0\end{cases}
$$

Proof. If $v_{P}(x)=0$, we have $v_{P}\left(D_{t_{P}} x\right) \geq 0$. Therefore $v_{P}\left(\frac{D_{t_{P}}^{i} x}{x}\right) \geq 0$ if $v_{P}(x)=0$.

If $v_{P}(f)=m \neq 0$, then there exists $\eta \in K$ with $v_{P}(\eta)=0$ such that $x=t_{P}^{m} \eta$.

By Proposition 1.1.a

$$
\begin{aligned}
\frac{D_{t_{P}}^{i} x}{x} & =\frac{1}{x} \sum_{j=0}^{i}\left(\begin{array}{c}
m \\
j
\end{array}\right) t_{P}^{m-j} D_{t_{P}}^{i-j} \eta \\
& =\frac{t_{P}^{-i}}{\eta} \sum_{j=0}^{i}\left(\begin{array}{c}
m \\
j
\end{array}\right) t_{P}^{i-j} D_{t_{P}}^{i-j} \eta .
\end{aligned}
$$

Therefore $v_{P}\left(\frac{D_{t_{P}}^{i} x}{x}\right) \geq-i$. 
Proposition 3.3. Let $L_{1}, \ldots, L_{q}$ be linear forms in $n+1$ variables with coefficients in $k$ and in general position. Let $x_{0}, \ldots, x_{n}$ be elements of $K$ such that $L_{i}\left(x_{0}, \ldots, x_{n}\right) \not \equiv$ 0. Denote by $l_{i}=L_{i}\left(x_{0}, \ldots, x_{n}\right)$. If $v_{P}\left(l_{1}\right) \leq v_{P}\left(l_{2}\right) \leq \ldots \leq v_{P}\left(l_{q}\right)$, then $v_{P}\left(l_{1}\right)=$ $v_{P}\left(l_{2}\right)=\ldots=v_{P}\left(l_{q-n}\right)=\min _{0 \leq i \leq n}\left\{v_{P}\left(x_{i}\right)\right\}$.

Proof. See [Wa], Proposition 4.2. $\square$

Theorem 2. Suppose that $x_{0}, \ldots, x_{n}$ are elements of $K$, and are linearly independent over $k K^{p^{m}}$. Suppose that $L_{1}, \ldots, L_{q}$ are linear forms in $n+1$ variables with coefficients in $k$, and are in general position, i.e. any $n+1$ elements of $\left\{L_{i}\right\}$ are linearly independent over $k$. Then there exists a sequence of numbers $0=\epsilon_{0}<\epsilon_{1} \cdots<\epsilon_{n}$, such that

$$
\begin{aligned}
(q-n-1) h\left(x_{0}, \ldots, x_{n}\right) & \leq \sum_{i=1}^{q} \sum_{P \notin S} \min _{1 \leq i \leq q}\left\{\epsilon_{n}, v_{P}\left(L_{i}\left(x_{0}, \ldots, x_{n}\right)\right)-\min _{0 \leq j \leq n}\left\{v_{P}\left(x_{j}\right)\right\}\right\} \\
& +\left(\epsilon_{1}+\cdots+\epsilon_{n}\right) \max \{0,2 g-2+|S|\}
\end{aligned}
$$

Furthermore, when the characteristic of $p=0, \epsilon_{i}=i$; when $p>0$, the upper bound for $\epsilon_{i}$ is described in Theorem 1.

Proof.

If $x_{0}, \ldots, x_{n}$ are linearly independent over $k K^{p^{m}}(p \geq 0)$, then by Theorem 1 and its remarks there exists a sequnce of bounded numbers $0=\epsilon_{0}<\cdots<\epsilon_{n}$ such that

$$
\operatorname{det}\left(D_{t}^{\epsilon_{i}} x_{j}\right) \neq 0
$$

Let $L_{i}\left(x_{0}, \ldots, x_{n}\right)=\sum_{j=0}^{n} a_{i j} x_{j}:=l_{i}$ for $1 \leq i \leq q$. Let $I=\left\{i_{0}, \ldots, i_{n}\right\}$ be an index subset of $\{1, \ldots, q\}$ and $\left\{u_{1}, \ldots, u_{q-n-1}\right\}$ be the complement of $I$. Let

$$
G=\frac{l_{1} \ldots l_{q}}{\operatorname{det}\left(D_{t}^{\epsilon_{i}} x_{j}\right)} .
$$

By Proposition 3.1.a

$$
\operatorname{det}\left(D_{t}^{\epsilon_{i}} l_{i_{j}}\right)=\operatorname{det}\left(a_{i j}\right) \operatorname{det}\left(D_{t}^{\epsilon_{i}} x_{j}\right)
$$

Then we have the following formula:

$$
\begin{aligned}
l_{u_{1}} \ldots l_{u_{q-n-1}} & =c_{I} G \frac{\operatorname{det}\left(D_{t}^{\epsilon_{i}} l_{i_{j}}\right)}{l_{i_{0}} \ldots l_{i_{n}}} \\
& =c_{I} G \operatorname{det}\left(\frac{D_{t_{P}}^{\epsilon_{i}} l_{i_{j}}}{\prod_{i=0}^{n} l_{i_{j}}}\right)\left(\frac{d t}{d t_{P}}\right)^{-\sum_{i=0}^{n} \epsilon_{i}},
\end{aligned}
$$


where $c_{I}$ is a nonzero constant in $k$. Let $e_{P}=-\min _{0 \leq i \leq n}\left\{v_{P}\left(x_{i}\right)\right\}$. By Proposition 3.1.b we also have

$$
v_{P}\left(\operatorname{det}\left(\frac{D_{t_{P}}^{\epsilon_{i}} l_{i_{j}}}{l_{i_{j}}}\right)\right)=v_{P}\left(\operatorname{det}\left(\frac{D_{t_{P}}^{\epsilon_{i}} L_{i_{j}}\left(t_{P}^{e_{P}} f_{0}, \ldots, t_{P}^{e_{P}} f_{n}\right)}{\prod_{i=0}^{n} L_{i_{j}}\left(t_{P}^{e_{P}} f_{0}, \ldots, t_{P}^{e_{P}} f_{n}\right)}\right)\right)
$$

Assume that $v_{P}\left(l_{1}\right) \geq v_{P}\left(l_{2}\right) \geq \cdots \geq v_{P}\left(l_{q}\right)$ for $P \in C$. Then by Proposition 3.3, $v_{P}\left(l_{n+1}\right)=\cdots=v_{P}\left(l_{q}\right)=\min \left\{v_{P}\left(x_{i}\right)\right\}=-e_{P}$. Let $I=\{1, \ldots, n+1\}$. Then $(3.3)$ and (3.4) imply

$$
(q-n-1) \min \left\{v_{P}\left(x_{i}\right)\right\}=v_{P}(G)+v_{P}\left(\operatorname{det}\left(\frac{D_{t_{P}}^{\epsilon_{i}}\left(l_{i_{j}} t^{e_{p}}\right)}{\prod_{i=0}^{n} l_{i_{j}} t^{e_{P}}}\right)\right)-\sum_{i=0}^{n} \epsilon_{i} v_{P}\left(\frac{d t}{d t_{P}}\right)
$$

By Proposition 3.2, we have the following two inequalities:

$$
\begin{gathered}
v_{P}\left(\operatorname{det}\left(\frac{D_{t_{P}}^{\epsilon_{i}}\left(l_{i_{j}} t^{e_{P}}\right)}{\prod_{i^{n} 0}^{n} l_{i_{j}} t^{e_{P}}}\right)\right) \geq-\min _{0 \leq i \leq n}\left\{\epsilon_{i}, v_{P}\left(l_{i_{j}}\right)+e_{P}\right\} . \\
v_{P}\left(\operatorname{det}\left(\frac{D_{t_{P}}^{\epsilon_{i}}\left(l_{i_{j}} t^{e_{P}}\right)}{\prod_{i=0}^{n} l_{i_{j}} t^{e_{P}}}\right)\right) \geq \sum_{i=0}^{n} \epsilon_{i} .
\end{gathered}
$$

Let $S$ be a finite set of points of $C$. Then (3.5), (3.6), (3.7) and the Riemann-Roch theorem (cf. [Ma]) imply

$$
\begin{aligned}
(q-n-1) h\left(x_{0}, \ldots, x_{n}\right) & \leq \sum_{P \notin S} \sum_{j=1}^{q} \min \left\{\epsilon_{n}, v_{P}\left(l_{j}\right)+e_{P}\right\} \\
& +\left(\epsilon_{1}+\cdots+\epsilon_{n}\right) \max \{0,2 g-2+|S|\} .
\end{aligned}
$$

This completes the proof.

\section{REFERENCES}

[B-M] Brownawell, D and Masser, D., Vanishing Sums in Function Fields, Math. Proc. Cambridge Philos. Soc. 100 (1986), 427-434.

[G-V] Garcia, A and Voloch, J. F., Wronskians and Linear Independence in Fields of Prime Charactereistic, Manuscripta Math. 59 (1987), 457-469.

[La] Lang, S., Introduction to Complex Hyperbolic Spaces, Springer-Verlag, 1987.

[Ma] Mason, R. C., Diophantine Equations over Function Fields LMS. Lecture Notes 96, Cambridge Univ. Press, 1984.

[No] Noguchi, J., Nevanlinna-Cartan Theory and a Diophantine Equation over Function Fields, J. rein angew. Math. 487 (1997), 61-83.

[S-V] Stöhr, K-O. and Voloch, J. F., Weierstrass Points and Curve over Function Fields, Proc. London Math. Soc.(3) 52 (1986), 1-19.

[Si] Silverman, J. H., The Arithmetic of Elliptic Curves, Springer-Verlag, 1985.

[Vol] Voloch, J. F., Diagonal Equations over Function Fields, Bol. Soc. Brazil Math. 16 (1985), 29-39.

[Wa1] Wang, J., T.-Y., The Truncated Second Main Theorem of Function Fields, J. of Number Theory 58 (1996), 139-157. 
[Wa2] Wang, J., T.-Y., S-integral points of $P^{n}-\{2 n+1$ hyperplanesingeneralposition $\}$ over number fields and function fields, Trans. Amer. Math. Soc. 348 (1996), 3379-3389.

[Wa3] Wang, J., T.-Y., Integral points of projective spaces omitting hyperplanes over function fields of positive characteristic, preprint 1997.

[Wa4] Wang, J., T.-Y., abc estimate, integral points, and geometry of $P^{n}$ minus hyperplanes, preprint 1998.

Institute of Mathematics, Academia Sinica, Nankang, Taipei 11529 Taiwan, R.O.C.

E-mail address: jwang@ math.sinica.edu.tw 